\title{
SANS Investigation of Valency Effect of Salts on Water/AOT/ Dodecane Microemulsion Droplets
}

\author{
Sohrab Abbas ${ }^{1, a}$, V. K. Aswal ${ }^{1,}$ and J. Kohlbrecher ${ }^{2}$ \\ ${ }^{1}$ Solid State Physics Division, Bhabha Atomic Research Centre, Mumbai 400 085, India \\ ${ }^{2}$ Laboratory for Neutron Scattering, Paul ScherrerInstitut, CH-5232 PSI Villigen, Switzerland \\ ${ }^{\text {a)} C o r r e s p o n d i n g ~ a u t h o r: ~ a b b a s @ b a r c . g o v . i n ~}$
}

\begin{abstract}
Small Angle Neutron Scattering (SANS) has been employed to demonstrate the formation and stabilization of water/AOT/dodecane microemulsion (water in oil) droplets. The droplet volume fraction $\varphi$ plays an important role in determining the microemulsion structure, interaction and in tuning the strength of inter-droplet attractive interaction. We demonstrate that the inter and intradroplet interfacial properties can be modified and controlled by using mono and multivalent salts. The structure and interaction has been modeled using modified Ornstein-Zernicke relation. The addition of monovalent $(\mathrm{NaCl})$ salt of $0.3 \mathrm{M}$ concentration to microemuslion system with varying droplet volume fraction significantly reduces the interdroplet interaction and increases the droplet stability. However, the role of mutlivalent ions are more complex and the electric repulsion comes into play while tuning the structure and interaction properties.
\end{abstract}

\section{INTRODUCTION}

Microemulsions have been widely studied due to its multitude practical applications in industries that include oil recovery, pharmacochemistry, paint and biological fields etc. This can largely be attributed to their unique properties such as small interfacial tension, large interfacial area, thermodynamic stability etc. [1-2]. A water in oil (w/o, reverse micelles) microemulsion is dispersion of small spherical water droplets in a continuous oil phase, surrounded by a layer of surfactant molecules. The rich phase behavior of the AOT stabilized w/o microemulsions has gained significant importance, in the last few decades due to its formation in absence of any co-surfactants. The roles of the oils, surfactants, temperature, and water soluble electrolytes in determining the rich phase behavior of these microemulsions have been carried out by many groups using different characterization techniques [3-5]. Any potential application requires the tuning of inetefacial interactions and the usage of smaller amount of AOT for environmental benefits. We have earlier carried out the studies to probe the stability and interaction of water/AOT/dodecane reverse microemulsion droplets using SANS measurements and investigated the effect of variation in the molar ratio of water to AOT on the microemulsion droplets with water volume fraction being kept constant. It was demonstrated that the inter droplet attractive interaction can be tuned by varying the salt concentration and system can have enhanced stability even at smaller AOT concentration [6].

The present work deals with the investigation of stabilization of microemsulsion droplet, structure and interaction as a function of droplet volume fraction using SANS measurements. It is shown that droplet volume fraction too plays vital role in determining the stability of microemuslison. It has been demonstrated that interdroplet interaction can effectively be controlled by tuning the interfacial droplet properties by employing multivalent salts. The Ionic strength of different multivalent salt has also been utilized to control the droplet structure and intra and interfacial droplet interactions.

\section{EXPERIMENTAL}

AOT (Sodium-2-diethylhexyl sulfosuccinate, $\geq 97 \%$ ) was purchased from Sigma-Aldrich. n-Dodecane $\left(\mathrm{C}_{12} \mathrm{H}_{26}\right)$ and electrolytes $\mathrm{NaCl}, \mathrm{CaCl}_{2}, \mathrm{AlCl}_{3}$, and $\mathrm{ZrCl}_{4}$ were obtained from Otto Chemie Pvt. Ltd., India. The samples were prepared by mixing the appropriate amount of components in glass vial and were tightly sealed. The 
droplet volume fraction $\varphi$ (= water droplet volume/ total volume) and the molar ratio $W$ of water $\left(\mathrm{D}_{2} \mathrm{O}\right)$ to surfactant molecules, $W=\left[\mathrm{D}_{2} \mathrm{O}\right] /[\mathrm{AOT}]$, determine the sample composition. A stock solution of $W 5(W \mathrm{~N}$, with $\mathrm{N}$ describing the value of sample's molar ratio of $\mathrm{D}_{2} \mathrm{O}$ to AOT e.g., here 5) was prepared by mixing the proper AOT amount in dodecane and $\mathrm{D}_{2} \mathrm{O}$. The samples with varying $\varphi$ were prepared by diluting the stock solution of $\varphi=0.2$. All samples were transparent at $30^{\circ} \mathrm{C}$. The samples were thoroughly shaken for several minutes to ensure homogenization and then kept at room temperature for several hours before the SANS experiment. The samples for SANS experiments were prepared in $\mathrm{D}_{2} \mathrm{O}$ to exploit the better scattering length density contrast.

SANS experiments were carried out at SANS-I facility, Swiss Spallation Neutron Source SINQ, Paul Scherrer Institut, Switzerland [7]. The instrument used a velocity selector to produce a monochromatic neutron beam of $6 \AA$ wavelength with a nominal wavelength spread of $10 \%$. Data were collected at two sample-to-detector distances 2 and 8 and to cover a wide wave vector transfer $\mathrm{Q} \sim 0.04-3.0 \mathrm{~nm}^{-1}$. The microemulsion samples were loaded in $2 \mathrm{~mm}$ thick Hellma quartz cell at fixed temperature of $30^{\circ} \mathrm{C}$.

\section{DATA ANALYSIS}

The measured coherent scattering intensity as a function of $Q(=4 \pi \sin \theta / \lambda ; 2 \theta$ is scattering angle, $\lambda$ is wave length of neutrons) in SANS experiments, can be represented by [8]

$$
I(Q)=\varphi V\left(\rho_{p}-\rho_{s}\right)^{2} P(Q) S(Q)+B
$$

where $\varphi$ denotes for the particles volume fraction and $V$ signifies particle volume. $\rho_{p}$ and $\rho_{\mathrm{s}}$ denote scattering length densities of particle and solvent, respectively. The terms $P(Q)$ and $S(Q)$ define particle form factor and interparticle structure factor, respectively. $B$ is a constant, signifying incoherent background. $P(Q)$ accounts for the scattering from a single particle and hence is a function of shape and size of the particle whereas the structure factor $S(Q)$ is Fourier transform of the radial distribution function $g(r)$ and hence yields the information concerning correlation between the particles present in the system. The expressions of $P(Q)$ for different shapes can be accessed in literature [8]. The interaction between the droplets can be expressed as a sum of attractive and repulsive forces. In this case, $S(Q)$ is ccomputed by considering hard sphere repulsive potential combined with a short range strong attraction using modified Ornstein-Zernicke relation [9]

$$
S(Q)=1+\frac{S(0)}{1+(Q \xi)^{2}}
$$

where $\xi$ represents the correlation length and $S(0)=n k_{B} T K$, with $\mathrm{n}$ being droplet number density, $k_{B}$ the Boltzmann constant, $T$ the temperature. $K$ denotes the isothermal compressibility.

\section{RESULTS AND DISCUSSION}

AOT is a doubly tailed anionic surfactant with negatively charged sulfonate head group attracted to water droplet and hydrophobic hydrocarbon chains aligned towards oil phase. Fig. 1a represents the SANS data of microemulsion system with increasing $\varphi(\varphi=0.01$ to 0.15$)$ at constant molar ratio of $\mathrm{D}_{2} \mathrm{O}$ to AOT, W15. As $\varphi$ increases, interdroplet attractive interaction increases which is manifested by the increase in the scattered intensity at lower Q. The inter-droplet attractive interaction in these w/o microemulsion becomes stronger progressively with increase in $\varphi$ and then start decreasing at high droplet volume fraction $\varphi$. We consider modified Ornstein-Zernicke model to explain the results and to extract the structure and interaction information of the microemulsion system. We consider a spherical form factor for scattering from droplet core $\left(\mathrm{D}_{2} \mathrm{O}\right)$ and a Lorentzian shaped inter-droplet interaction $\mathrm{S}(\mathrm{Q})$ as given by Eq. 2 to account for interactions. The fitted parameters are droplet size, polydispersity, $S(0)$ and correlation length $\xi$. The correlation length and compressibility which is proportional to $\mathrm{S}(0)$ increase simultaneously and then $\mathrm{S}(0)$ start decreasing at higher $\varphi$ (Table 1, Fig. 1b). The structure factor $S(Q)$ increases monotonically along with the $P(Q)$ upto $\varphi=0.05$, then remains constant upto 0.1 and decreases afterwards. At smaller values of $\varphi$ 's, the $S(Q)$ is only slightly greater than unity, indicating the absence of any strong interdroplet attraction/correlation and the formation of stable droplets due to large AOT faction vis á vis $\mathrm{D}_{2} \mathrm{O}$ droplets. For larger $\varphi$, the droplet fraction becomes large leading to decrease in microemulsion droplets and increase in $S(Q)$ implying the increase in droplet attraction. This can be attributed to volume exclusion effect (steric repulsion, entropic origin). The parameter $\varphi$ plays important role in controlling the size of the droplets as well as stability. 

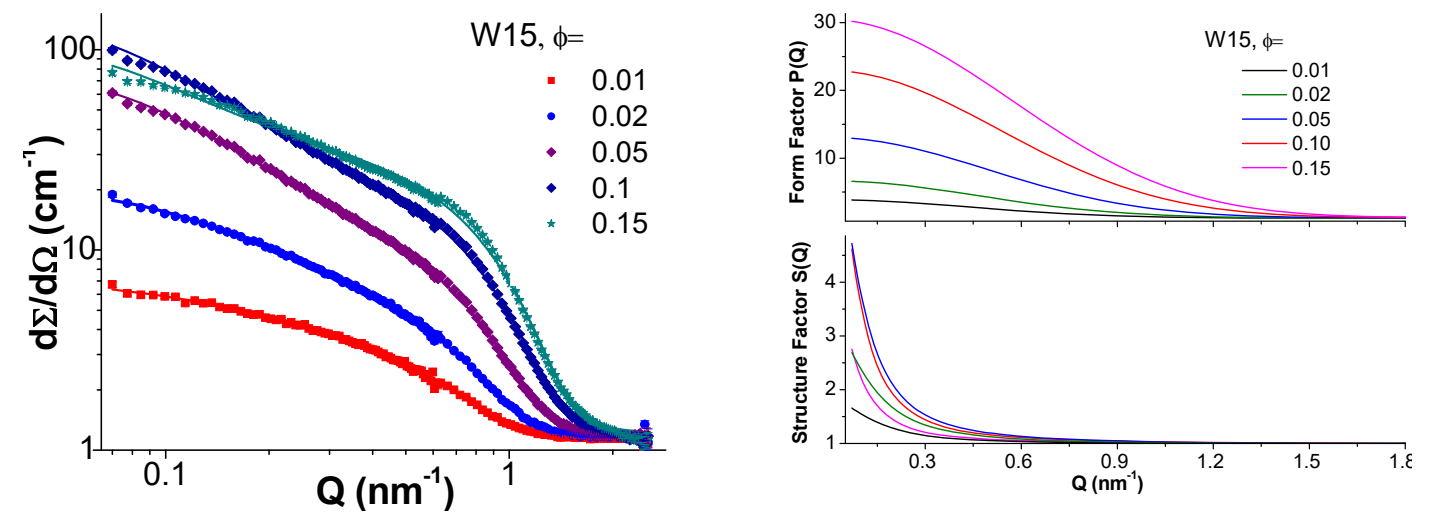

FIGURE 1. SANS data of microemulsion system with varying volume fraction of AOT to total (a) and the fitted form factor and structure factor as a function of $Q(\mathrm{~b})$.

The repulsion between anionic sulfonate head group on droplet surface restrain other head group to come closer on droplet surface, consequently suppressing the strength of steric repulsion. To tune the AOT head group repulsion and stabilize (increase in steric repulsion) the system, $0.3 \mathrm{M}$ salt $(\mathrm{NaCl})$ was added to varying $\varphi(=0.01$ to 0.15$)$ [Fig. 2a]. The presence of salt screens the AOT head group repulsion, reducing the interfacial repulsion between AOT head groups allowing more of these head groups on droplet surface and inducing them to come closer to droplet $\mathrm{D}_{2} \mathrm{O}$ core. The $S(Q)$ reduces to $\sim 1$ and also $\xi$ becoming smaller indicating the disappearance of inter droplet attractive interaction. This increases droplet stability by reducing the chances of droplet coalescence and aggregation (Fig. 2). As salt not only reduces the water core radius but also causes the reduction in polydispersity, we investigated the effectiveness of various salts of different valencies on stabilization of microemulsion droplets by adding salts of $0.3 \mathrm{M} \mathrm{NaCl}, 0.1 \mathrm{M} \mathrm{CaCl}_{2}, 0.3 \mathrm{M} \mathrm{AlCl}_{3}$ and $0.1 \mathrm{M} \mathrm{ZrCl}_{4}$. Fig. 3(a) depicts the SANS measurements of microemulsion systems with different salta and their fitted form factor and structure factor. On adding salts, the scattered intensity gets smaller at lower $\mathrm{Q}$ values for $\mathrm{NaCl}, \mathrm{CaCl}_{2}, \mathrm{AlCl}_{3}$ indicating the formation of more stable structure compared to no salt droplets due to reduced intra-droplet interfacial interaction. However, scattered intensity gets up turn at smaller Q for $\mathrm{ZrCl}_{4}$ implying less stable structure and increased intra-droplet interfacial interaction. $S(Q)$ deviates significantly from that of $\mathrm{NaCl}(S(Q \sim 1)$ for multivalent ions and becomes even greater than the value of no salt for $\mathrm{ZrCl}_{4}$ due to increased correlation length and compressibility indicating the large increase in attraction between droplets [Fig. 3(b)]. It is noted that higher-valent salts in added concentrations compared to $\mathrm{NaCl}$ were less effective in stabilizing the structure due to their larger ionic strength and resultant electric repulsion between multivalent ions.
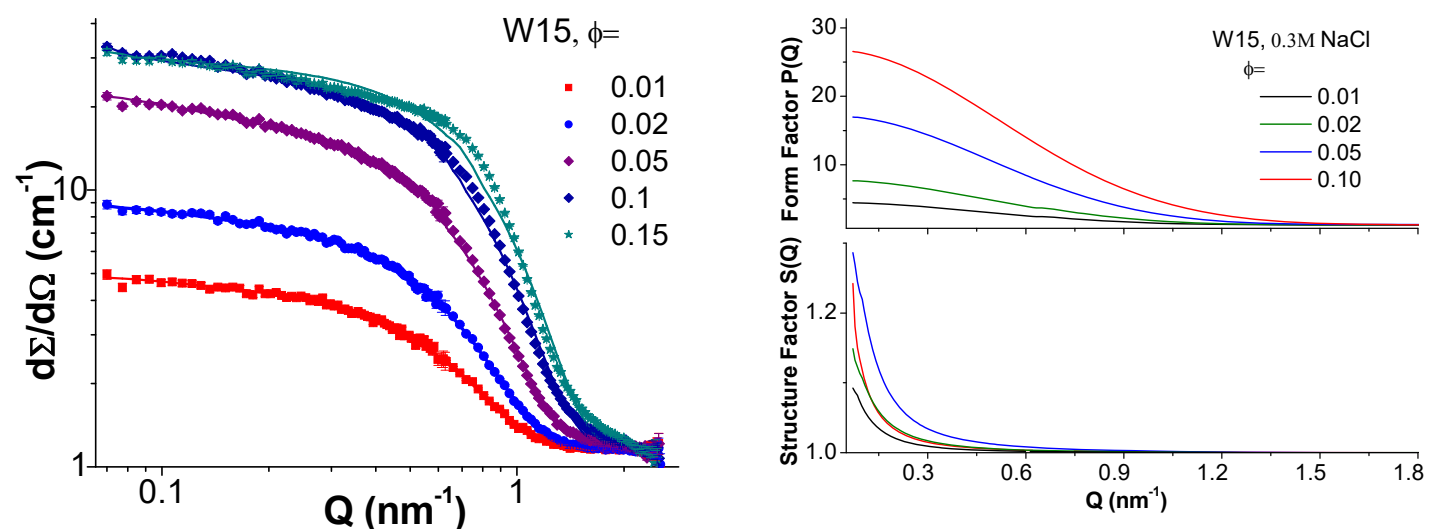

FIGURE 2. SANS data of microemulsion system with varying $\varphi$ at a fixed salt concentration of $0.3 \mathrm{M}$ NAcl (a) and the fitted form factor and structure factor as a function of $Q(\mathrm{~b})$. 
TABLE 1. Fitted parameters of SANS data with Ornstein-Zernicke model for fixed $W 15$ AOT/water/dodecane microemulsions with varying volume droplet fraction $\varphi=0.01-0.1$

\begin{tabular}{|ccccc|}
$\begin{array}{c}\text { Micro } \\
\text { Emulsion } \phi\end{array}$ & $\begin{array}{c}\text { Droplet } \\
\text { radius } \boldsymbol{R}(\mathbf{n m})\end{array}$ & $\begin{array}{c}\text { Polydisp } \\
\text { ersity }\end{array}$ & $\boldsymbol{S ( 0 )}$ & $\xi(\mathbf{n m})$ \\
0.01 & 3.05 & 0.21 & 1.14 & 6.66 \\
0.02 & 2.96 & 0.19 & 2.60 & 7.46 \\
0.05 & 2.79 & 0.19 & 6.18 & 10.23 \\
0.10 & 2.64 & 0.18 & 6.47 & 11.99 \\
0.15 & 2.33 & 0.16 & 3.15 & 12.19 \\
\hline
\end{tabular}

TABLE 2. Fitted parameters of SANS data with Ornstein-Zernicke model for fixed $W 15, \varphi=0.1$ AOT/water/dodecane microemulsions with differnt salts of different ionic strength

$\begin{array}{|ccccc|}\text { Salt } & \text { Droplet radius } \boldsymbol{R}(\mathbf{n m}) & \text { Polydispersity } & \boldsymbol{S}(\mathbf{0}) & \boldsymbol{\xi}(\mathbf{n m}) \\ \mathrm{No} \mathrm{Salit} & 2.64 & 0.18 & 6.47 & 11.99 \\ 0.3 \mathrm{M} \mathrm{NaCl} & 2 . .68 & 0.19 & 0.69 & 13.64 \\ 0.1 \mathrm{M} \mathrm{CaCl} & 2.67 & 0.17 & 1.59 & 9.95 \\ 0.3 \mathrm{M} \mathrm{AlCl}_{3} & 2.59 & 0.17 & 3.02 & 10.03 \\ 0.1 \mathrm{M} \mathrm{ZrCl} & 2.33 & 0.15 & 11.4 & 15.46\end{array}$
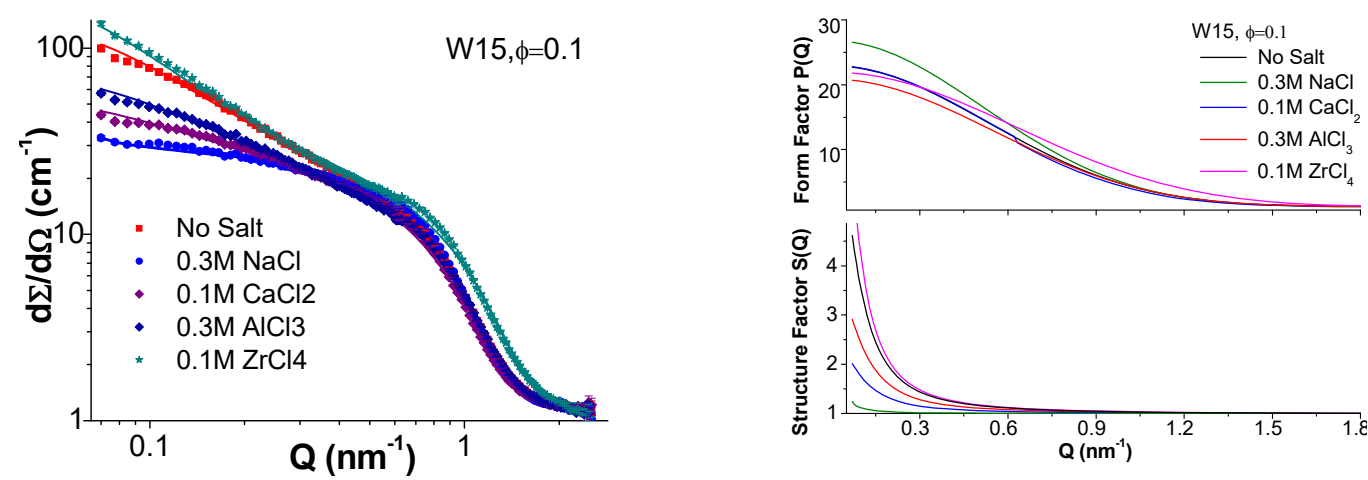

FIGURE 3. SANS data of microemulsion system with different mono and multivalent salts at fixed W15 and $\varphi=0.1$ (a) and the fitted form factor and structure factor as a function of $Q(\mathrm{~b})$.

To conclude, the formation and stabilization of water/AOT/dodecane microemulsion droplets using SANS has been demonstarted. The strengthening of the inter-droplet attractive interaction with increase in $\varphi$ has been modeled using modified Ornstein-Zernicke relation. $\varphi$ plays significant role in determining the microemulsion structure, interaction and in tuning the strength of inter-droplet attractive interaction. It has been shown that inter and intradroplet interfacial properties can be modified and controlled by using mono and multivalent salts. The addition of monovalent $(\mathrm{NaCl})$ salt of $0.3 \mathrm{M}$ concentration to microemuslion system with varying droplet fraction significantly reduces the interdroplet interaction and increases the droplet stability. However, Mutlivalent ions plays complex role due to appearance of electric repulsion while tuning the structure and interaction properties. The Ionic strength of multivalent salt can effectively be tuned to control the droplet structure and intra and interfacial droplet interactions.

\section{REFERENCES}

1. J. H. Schulman, W. Stoekenius, L.M.Prince, J. Phys. Chem. 63, 1677-1680 (1959).

2. P. L. Luisi, L. J. Majid, J. H. Fendler,. Crit. Rev. Biochem. 20, 409-474 (1986).

3. C. Cametti, P. Codastefano, P. Tartaglia, J. Rouch, S.H. Chen, Phys. Rev. Lett. 64 (1990) 1461.

4. A. D. Dinsmore, M. F. Hsu, M. G. Nikolaides, M. Marquez, A. R. Bausch and D. A. Weitz, Science 298, 1006-1009 (2002).

5. A. Kogan, A.N. Garti, Adv. Colloid Interface Sci. 123-126 (2006) 369.

6. Sohrab Abbas, D Saha, and V. K. Aswal AIP Conference Proceedings 2115, 030034: 1-4 (2019)

7. J. Kohlbrecher and W. Wagner, J. Appl. Crystallogr. 33, 804 (2000).

8. J. S. Pedersen, Adv. Coll. Inter. Sci.70, 171-210 (1997).

9. E. W Keller, J. F. Billman, J. L. Fulton and R. D. Smith, J. Phys. Chem. 95, 458-462 (1991). 